\title{
Some consequences of the inequality conditions in contact and crack problems
}

\section{J. DUNDURS}

Department of Civil Engineering, Northwestern University, Evanston, Ilinois 60201, U.S.A.

\section{MARIA COMNINOU}

Department of Applied Mechanics and Engineering Science, University of Michigan, Ann Arbor, Michigan 48109, U.S.A.

(Received March 1, 1978)

\begin{abstract}
The importance of the inequalities and related side conditions that must be incorporated in contact and crack problems is emphasized and the ensuing consequences explored. An asymptotic analysis of the transitions from slip to separation, stick to slip, and stick to separation is carried out. The inequalities in contact problems make the contact pressure continuous for all levels of friction. They also make a direct transition from stick to separation impossible, unless the combination of materials is special. The inequalities in crack problems are less stringent, but they preclude certain singularities that appear to have flourished in the literature previously.
\end{abstract}

\section{RÉSUMÉ}

On appuie sur l'importance d'inéquations et d'autres conditions auxiliaires qui doivent être comprises dans les problèmes de contact et de fissure, et on en explore les conséquences. On emploie une analyse asympotique sur les transitions entre les zones glissement-décollement, adhérence-glissement et adhérence-décollement. Il s'ensuit que la contrainte normale du contact doit être continue pour toutes les valeurs du frottement. De plus, la transition directe adhérence-décollement est impossible, à moin que la combinaison des matériaux ne soit exceptionelle. Les inéquations dans les problèmes de fissure sont moins fortes, mais elles sont cependant suffisantes pour empêcher l'existence de certaines singularités qui apparaissent souvent dans les études précedentes.

\section{Introduction}

There are three possible transitions in the boundary conditions of contact problems: slip to separation, stick to slip, and stick to separation. The last two types can also occur in crack problems. Thus the transition from stick to slip is similar to that from an undamaged bond to a closed tip of a crack, and stick to separation corresponds to undamaged bond or an adhesion zone changing to an open tip of the crack. An abrupt change in boundary conditions generally leads to singularities. The question we explore is how the elastic fields near a transition are locally restricted by the inequalities that must be incorporated in contact and crack problems. In either case, 
the material cannot be allowed to overlap, and the requirement is that the gap between the solids be non negative. The additional conditions in contact problems are that the normal tractions transmitted between the solids cannot be tensile, and that the tangential tractions are limited by the available friction.

The analysis we perform is quite simple. It is based on the Williams technique [1, 2] for finding the asymptotic nature of the elastic fields near a transition in the boundary conditions. The problem is particularly easy to set up using the catalogue given in the Appendix of [3]. The intermediate algebra may be tedious, but we do not think it necessary to give any of the details, and thus we merely state and interpret the results.

The placement of the coordinate axes in relation to the two elastic solids and the different zones are shown in Figure 1. The quantities entering the boundary conditions are the gap between the two bodies

a)

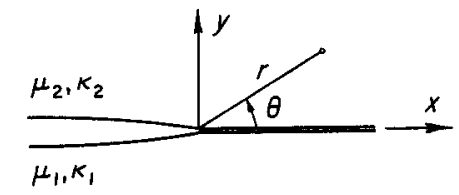

b)

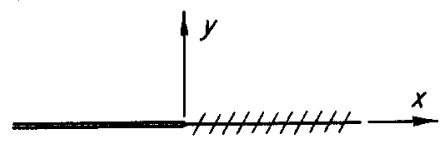

c)

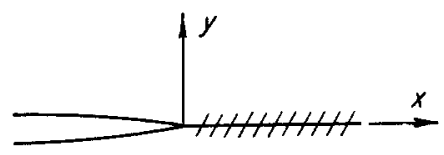

Figure 1

$$
g(x)=u_{y}^{(2)}(x, 0)-u_{y}^{(1)}(x, 0)
$$

the tangential shift

$$
h(x)=u_{x}^{(2)}(x, 0)-u_{x}^{(1)}(x, 0)
$$

the normal traction

$$
N(x)=\sigma_{y y}^{(1)}(x, 0)=\sigma_{y y}^{(2)}(x, 0)
$$

and the tangential or shear traction

$$
T(x)=\sigma_{x y}^{(1)}(x, 0)=\sigma_{x y}^{(2)}(x, 0) .
$$

Note that the requirement of the tractions being continuous at the interface is already incorporated in (1.3) and (1.4).

The boundary conditions in the separation zone for both contact and crack problems are

$$
N(x)=0, \quad T(x)=0
$$




$$
g(x) \geq 0 \text {. }
$$

In the slip zone, the boundary conditions are considerably more complicated:

$$
\begin{array}{ll}
g(x)=0 ; & |T(x)|=f|N(x)| \\
N(x) \leq 0 ; & \operatorname{sgn} T(x)=\operatorname{sgn} \dot{h}(x) .
\end{array}
$$

where $f=$ const is the coefficient of kinetic friction. Equation (1.9) shows that our considerations will be based on the simplest possible model for friction between two solids. The last of these conditions, or (1.11), may require some explanation. As pointed out first by Goodman [4], contact problems with friction generally require an incremental formulation. This is due to the fact that the deformations depend not only on the instantaneous values of the applied forces, but also are affected by the history of loading. There is no essential difference, however, between an incremental formulation and viewing the deformations to develop in time. Thus $\dot{h}(x)$ denotes $d h(x) / d t$, and (1.11) is nothing else than a condition that the sign of the tangential tractions be consistent with the direction of slip at any given instant.

The boundary conditions in the stick zone are

$$
\begin{array}{ll}
g(x)=0 ; & \dot{h}(x)=0 \\
N(x) \leq 0 ; & |T(x)|<f_{s}|N(x)|
\end{array}
$$

where $f_{s}\left(f_{s} \geq f\right)$ denotes the coefficient of static friction. The counterpart of these boundary conditions which must be satisfied in the adhesion zone of a crack problem are

$$
g(x)=0 ; \quad h(x)=0 .
$$

It may be noted that (1.1) is written as if the contact were receding [5, 6]. For an advancing contact, (1.1) must be replaced with

$$
g(x)=g_{0}(x)+u_{y}^{(2)}(x, 0)-u_{y}^{(1)}(x, 0)
$$

where $g_{0}(x)$ is the initial gap. However, if the initial gap is sufficiently smooth, $g_{0}(x)$ does not enter the asymptotic analysis. A similar comment applies also for cracks under internal loading.

\section{Transition from slip to separation}

Referring to Figure 1a, we consider the boundary conditions

$$
\begin{array}{ll}
u_{\theta}^{(1)}(r, 0)=u_{\theta}^{(2)}(r, 0) ; & \sigma_{r \theta}^{(1)}(r, 0)=\sigma_{r \theta}^{(2)}(r, 0) \\
\sigma_{\theta \theta}^{(1)}(r, 0)=\sigma_{\theta \theta}^{(2)}(r, 0) ; & \sigma_{r \theta}^{(1)}(r, 0)=-\rho \sigma_{\theta \theta}^{(1)}(r, 0) \\
\sigma_{r \theta}^{(1)}(r,-\pi)=\sigma_{r \theta}^{(2)}(r, \pi)=0 & \\
\sigma_{\theta \theta}^{(1)}(r,-\pi)=\sigma_{\theta \theta}^{(2)}(r, \pi)=0 .
\end{array}
$$

Both directions of slip can be taken into account by allowing the constant $\rho$ to assume also negative values. Thus $\rho=f$ corresponds to the upper solid slipping with 
respect to the lower body in the positive $x$-direction, while $\rho=-f$ indicates slip in the opposite direction.

The determinant of the coefficients which must vanish for a nontrivial solution and thus yields the possible deformation states in the vicinity of the transition point is [7, 8]

$$
\Delta(\lambda)=\sin ^{3} \lambda \pi(\cos \lambda \pi+\rho \beta \sin \lambda \pi)
$$

where

$$
\beta=\frac{\mu_{2}\left(\kappa_{1}-1\right)-\mu_{1}\left(\kappa_{2}-1\right)}{\mu_{2}\left(\kappa_{1}+1\right)+\mu_{1}\left(\kappa_{2}+1\right)} .
$$

If the strain energy density is to be integrable, we must have $\lambda<1$.

One set of the roots of (2.9) is determined by

$$
\cot \lambda \pi=-\rho \beta \text {. }
$$

The elastic fields corresponding to these roots are

$$
\begin{aligned}
2 \mu u_{\theta}^{(1)}= & \frac{C}{2(1-\lambda)} r^{1-\lambda}\left\{\left(1+\rho^{2} \beta\right)\left(\kappa_{1}-1+\lambda\right) \cos \lambda \theta-\left[\lambda-\rho^{2} \beta(2-\lambda)\right] \cos (2-\lambda) \theta\right. \\
& \left.+\rho(1-\beta)\left(\kappa_{1}-1+\lambda\right) \sin \lambda \theta-\rho[2-\lambda(1-\beta)] \sin (2-\lambda) \theta\right\} \\
2 \mu_{1} u_{\theta}^{(1)}= & \frac{C}{2(1-\lambda)} r^{1-\lambda}\left\{-\left(1+\rho^{2} \beta\right)\left(\kappa_{1}+1-\lambda\right) \sin \lambda \theta+\left[\lambda-\rho^{2} \beta(2-\lambda)\right] \sin (2-\lambda) \theta\right. \\
& \left.\quad+\rho(1-\beta)\left(\kappa_{1}-1+\lambda\right) \cos \lambda \theta-\rho[2-\lambda(1-\beta)] \cos (2-\lambda) \theta\right\} \\
\sigma_{r r}^{(1)}= & \frac{1}{2} C r^{-\lambda}\left\{\left(1+\rho^{2} \beta\right)(2+\lambda) \cos \lambda \theta-\left[\lambda-\rho^{2} \beta(2-\lambda)\right] \cos (2-\lambda) \theta\right. \\
& +\rho(1-\beta)(2+\lambda) \sin \lambda \theta-\rho[2-\lambda(1-\beta)] \sin (2-\lambda) \theta\} \\
\sigma_{r \theta}^{(1)}= & \frac{1}{2} C r^{-\lambda}\left\{\left(1+\rho^{2} \beta\right) \lambda \sin \lambda \theta+\left[\lambda-\rho^{2} \beta(2-\lambda)\right] \sin (2-\lambda) \theta\right. \\
& -\rho(1-\beta) \lambda \cos \lambda \theta-\rho[2-\lambda(1-\beta)] \cos (2-\lambda) \theta\} \\
\sigma_{\theta \theta}^{(1)}= & \frac{1}{2} C r^{-\lambda}\left\{\left(1+\rho^{2} \beta\right)(2-\lambda) \cos \lambda \theta+\left[\lambda-\rho^{2} \beta(2-\lambda)\right] \cos (2-\lambda) \theta\right. \\
& +\rho(1-\beta)(2-\lambda) \sin \lambda \theta+\rho[2-\lambda(1-\beta)] \sin (2-\lambda) \theta\}
\end{aligned}
$$

where $C$ is a free constant. The expressions for the upper solid can be written from (2.12) to (2.16) by replacing $\mu_{1}$ with $\mu_{2}, \kappa_{1}$ with $\kappa_{2}$ and $\beta$ with $-\beta$. It may be noted that, while friction also affects the symmetric parts of the fields, the antisymmetric parts are entirely due to friction and vanish for $\rho=0$. In the slip zone we have

$$
\begin{array}{ll}
x>0 ; & h(x)=J C r^{1-\lambda} \\
& N(x)=C r^{-\lambda} ; \quad T(x)=-C \rho r^{-\lambda}
\end{array}
$$

and in the separation zone

$$
x<0: \quad g(x)=K C r^{1-\lambda} \sin \lambda \pi
$$


where

$$
\begin{aligned}
& J=\frac{1}{4(1-\lambda)}\left\{\frac{\kappa_{2}-1}{\mu_{2}}-\frac{\kappa_{1}-1}{\mu_{1}}-\rho^{2} \beta\left(\frac{\kappa_{2}+1}{\mu_{2}}+\frac{\kappa_{1}+1}{\mu_{1}}\right)\right\} \\
& K=\frac{1+\rho^{2} \beta^{2}}{4(1-\lambda)}\left(\frac{\kappa_{1}+1}{\mu_{1}}+\frac{\kappa_{2}+1}{\mu_{2}}\right)>0 .
\end{aligned}
$$

The other set of roots of (2.9) corresponds to $\sin \lambda \pi=0$

and, for $\lambda<1$, these roots lead to polar stress components that are either independent of $r$ or contain positive integer powers of $r$ :

$$
\begin{gathered}
2 \mu_{1} u_{r}^{(1)}=r^{1-\lambda}\left\{A\left[\left(\kappa_{1}-1+\lambda\right) \cos \lambda \theta+(2-\lambda) \cos (2-\lambda) \theta\right]\right. \\
\left.+B(1-\alpha)\left[\left(\kappa_{1}-1+\lambda\right) \sin \lambda \theta+\lambda \sin (2-\lambda) \theta\right]\right\} \\
2 \mu_{1} u_{\theta}^{(1)}=r^{1-\lambda}\left\{-A\left[\left(\kappa_{1}+1-\lambda\right) \sin \lambda \theta+(2-\lambda) \sin (2-\lambda) \theta\right]\right. \\
\left.+B(1-\alpha)\left[\left(\kappa_{1}+1-\lambda\right) \cos \lambda \theta+\lambda \cos (2-\lambda) \theta\right]\right\} \\
\begin{array}{r}
\sigma_{r r}^{(1)}=(1-\lambda) r^{-\lambda}\{A[(2+\lambda) \cos \lambda \theta+(2-\lambda) \cos (2-\lambda) \theta] \\
+B(1-\alpha)[(2+\lambda) \sin \lambda \theta+\lambda \sin (2-\lambda) \theta]\} \\
\sigma_{r \theta}^{(1)}=(1-\lambda) r^{-\lambda}\{A[\lambda \sin \lambda \theta-(2-\lambda) \sin (2-\lambda) \theta] \\
+B(1-\alpha) \lambda[-\cos \lambda \theta+\cos (2-\theta)]\} \\
\sigma_{\theta \theta}^{(1)}=(1-\lambda) r^{-\lambda}\{A(2-\lambda)[\cos \lambda \theta-\cos (2-\lambda) \theta] \\
+B(1-\alpha)[(2-\lambda) \sin \lambda \theta-\sin (2-\lambda) \theta]\}
\end{array}
\end{gathered}
$$

where $A$ and $B$ are free constants and

$$
\alpha=\frac{\mu_{2}\left(\kappa_{1}+1\right)-\mu_{1}\left(\kappa_{2}+1\right)}{\mu_{2}\left(\kappa_{1}+1\right)+\mu_{1}\left(\kappa_{2}+1\right)} .
$$

The corresponding expressions for the upper material follow by replacing $\mu_{1}$ with $\mu_{2}, \kappa_{1}$ with $\kappa_{2}, \alpha$ with $-\alpha$ and $A$ with a different free constant, say $A^{\prime}$. The components of these fields in cartesian coordinates are simply polynomials in $x$ and $y$. The root $\lambda=0$ gives the following constant stress fields:

$$
\begin{array}{ll}
\sigma_{x x}^{(1)}=4 A ; & \sigma_{x y}^{(1)}=\sigma_{y y}^{(1)}=0 \\
\sigma_{x x}^{(2)}=4 A^{\prime} ; & \sigma_{x y}^{(2)}=\sigma_{y y}^{(2)}=0 .
\end{array}
$$

It follows from the expressions above that

$$
\begin{aligned}
x>0: & & h(x)=L r^{1-\lambda} \\
& & N(x)=T(x)=0 \\
x<0: & & g(x)=0
\end{aligned}
$$


for all $\lambda$ satisfying (2.23). In (2.34)

$$
L=\frac{1}{2}\left(A^{\prime} \frac{\kappa_{2}+1}{\mu_{2}}-A^{\frac{\kappa_{1}+1}{\mu_{1}}}\right) \text {. }
$$

We enquire first whether it is possible to have a stress singularity at the transition point. As seen from the sets of expressions given for the stress components, a singularity can arise only from the root $0<\lambda_{0}<1$ of (2.11). In such case, $\sin \lambda_{0} \pi>0$ and, as seen from (2.18) and (2.20), to a positive gap in the separation zone correspond tensile normal tractions in the slip zone. Hence it is not possible to satisfy (1.7) and (1.10), and the free constant multiplying the singular terms must vanish.

Thus the two leading terms in the asymptotic expansions correspond to the root $\lambda=0$ of (2.23) and the root $\lambda_{0}-1$ of (2.11). These roots yield

$$
\begin{array}{ll}
x>0: & h(x)=U+L r+J C r^{2-\lambda_{0}} \\
& N(x)=C r^{1-\lambda_{0}} \\
& T(x)=-C \rho r^{1-\lambda_{0}} \\
x<0: & g(x)=-K C r^{2-\lambda_{0}} \sin \lambda_{0} \pi .
\end{array}
$$

The term $U=$ const in (2.38) arises from a possible rigid body displacement. The remaining conditions to be imposed are (1.7), (1.9), (1.10), and (1.11). Thus (1.7) and (1.10) give

$$
C \leq 0
$$

whereas (1.9) reduces to

$$
|\rho|=f \text {. }
$$

Using the dominant term in $h(x),(1.11)$ leads to

$$
\operatorname{sgn}(\rho)=\operatorname{sgn}(\dot{U}) \text {. }
$$

An important observation from (2.39) is that $N(0)=0$.

The final conclusion we can reach now is that, regardless of the value of the friction coefficient, the contact pressure at the transition from a slip zone to a separation zone is not only bounded, but must in fact vanish. Moreover, the asymptotic expansion of the contact pressure contains only fractional powers of $r$ determined by (2.11) in the range $\lambda<0$.

\section{Transition from stick to slip}

Placing the coordinate axes as shown in Figure $1 \mathrm{~b}$, we impose the following boundary conditions:

$$
\begin{array}{ll}
u_{r}^{(1)}(r, 0)=u_{r}^{(2)}(r, 0) ; & u_{\theta}^{(1)}(r, 0)=u_{\theta}^{(2)}(r, 0) \\
\sigma_{r \theta}^{(1)}(r, 0)=\sigma_{r \theta}^{(2)}(r, 0) ; & \sigma_{\theta \theta}^{(1)}(r, 0)=\sigma_{\theta \theta}^{(2)}(r, 0)
\end{array}
$$




$$
\begin{array}{ll}
u_{\theta}^{(1)}(r,-\pi)=u_{\theta}^{(2)}(r, \pi) ; & \sigma_{\theta \theta}^{(1)}(r,-\pi)=\sigma_{\theta \theta}^{(2)}(r, \pi) \\
\sigma_{r \theta}^{(1)}(r,-\pi)=\sigma_{r \theta}^{(2)}(r, \pi) ; & \sigma_{r \theta}^{(1)}(r,-\pi)=-\rho \sigma_{\theta \theta}^{(1)}(r,-\pi)
\end{array}
$$

where $\rho$ is a constant that will be related to the friction coefficient.

The determinant which must vanish for a nontrivial solution for the coefficients in the asymptotic analysis is [6]

$$
\Delta(\lambda)=\sin ^{3} \lambda \pi(\cos \lambda \pi-\rho \beta \sin \lambda \pi) .
$$

The roots of (3.9) which satisfy

$$
\cot \lambda \pi=\rho \beta
$$

lead to the following elastic fields:

$$
\begin{aligned}
& 2 \mu_{1} u_{r}^{(1)}=\frac{C}{2(1-\lambda)} r^{1-\lambda}\left\{\left(\kappa_{1}-1+\lambda\right)(1-\beta) \sin \lambda \theta-[2-\lambda(1-\beta)] \sin (2-\lambda) \theta\right\} \\
& 2 \mu_{1} u_{\theta}^{(1)}=\frac{C}{2(1-\lambda)} r^{1-\lambda}\left\{\left(\kappa_{1}+1-\lambda\right)(1-\beta) \cos \lambda \theta-[2-\lambda(1-\beta)] \cos (2-\lambda) \theta\right\} \\
& \sigma_{r r}^{(1)}=\frac{1}{2} C r^{-\lambda}\{(2+\lambda)(1-\beta) \sin \lambda \theta-[2-\lambda(1-\beta)] \sin (2-\lambda) \theta\} \\
& \sigma_{r \theta}^{(1)}=-\frac{1}{2} C r^{-\lambda}\{\lambda(1-\beta) \cos \lambda \theta+[2-\lambda(1-\beta)] \cos (2-\lambda) \theta\} \\
& \sigma_{\theta \theta}^{(1)}=\frac{1}{2} C r^{-\lambda}\{(2-\lambda)(1-\beta) \sin \lambda \theta+[2-\lambda(1-\beta)] \sin (2-\lambda) \theta\} .
\end{aligned}
$$

The corresponding expressions for the upper solid can be obtained by interchanging the elastic constants as done in connection with (2.12) to (2.16). It may be noted that the elastic fields given above are in the sense antisymmetric with respect to the interface. The quantities of particular interest that follow from these fields are

$$
\begin{array}{ll}
x>0: & N(x)=0 ; \quad T(x)=-C r^{-\lambda} \\
x<0 ; & h(x)=-M C r^{-\lambda} \sin \lambda \pi \\
& N(x)=C \beta r^{-\lambda} \sin \lambda \pi \\
& T(x)=-C r^{-\lambda} \cos \lambda \pi=-C \rho \beta r^{-\lambda} \sin \lambda \pi
\end{array}
$$

where

$$
M=\frac{1}{4(1-\lambda)}\left\{\frac{1}{\mu_{1}}\left[\left(\kappa_{1}-1\right)(1-\beta)+2\right]+\frac{1}{\mu_{2}}\left[\left(\kappa_{2}-1\right)(1+\beta)+2\right]\right\}>0 .
$$

The roots of (3.9) that satisfy

$\sin \lambda \pi=0$

lead to polar stress components that are independent of $r$ or contain positive integer powers of $r$. In cartesian coordinates, the elastic fields are simply polynomials in $x$ and $y$. Of interest for our purposes are only the stresses corresponding to $\lambda=0$ :

$$
\begin{aligned}
& \sigma_{x x}^{(1)}=A(1-\alpha)+B \frac{\alpha-2 \beta}{1+\alpha} ; \quad \sigma_{x x}^{(2)}=A(1+\alpha)-B \frac{\alpha-2 \beta}{1-\alpha} \\
& \sigma_{x y}^{(1)}=\sigma_{x y}^{(2)}=-B \rho ; \quad \sigma_{y y}^{(1)}=\sigma_{y y}^{(2)}=B
\end{aligned}
$$


and the associated results

$$
h(x)=0 ; \quad N(x)=B ; \quad T(x)=-B \rho
$$

valid for both $x>0$ and $x<0$.

We consider first the root $0<\lambda_{0}<1$ of $(3.10)$ which yields singular stresses. On basis of (3.16) and (3.17) it is immediately clear, however, that (1.15) cannot be satisfied, and we must conclude that no singular stresses are possible at the transition from stick to slip in contact problems.

Having discarded the first term in the asymptotic expansion, we study together the next two terms that correspond to the root $\lambda=0$ of (3.22) and the root $\lambda_{0}-1$ of (3.10). They lead to

$$
\begin{array}{ll}
x>0: & N(x)=B ; \quad T(x)=-B \rho-C r^{1-\lambda_{0}} \\
x<0: & h(x)=M C r^{1-\lambda_{0}} \sin \lambda_{0} \pi \\
& N(x)=B-C \beta r^{1-\lambda_{0}} \sin \lambda_{0} \pi \\
& T(x)=-B \rho+C \rho \beta r^{1-\lambda_{0}} \sin \lambda_{0} \pi .
\end{array}
$$

It may be noted that (1.8), (1.12), and (1.13) are satisfied on account of the boundary conditions imposed at the beginning. The conditions (1.14) and (1.15) imply that

$$
B<0
$$

and $(1.10)$ is also satisfied. Since $B<0$, the contact pressure cannot vanish at the transition from stick to slip. Equation (1.15) also implies that

$$
|\rho|<f_{s} .
$$

The last conditions to be considered are (1.9) and (1.11). Noting that $\sin \lambda \pi<0$, (1.11) yields

$$
\operatorname{sgn}(\rho)=\operatorname{sgn}(\dot{h})=\operatorname{sgn}(\dot{C})
$$

and (1.9) is satisfied by choosing

$$
|\rho|=f \text {. }
$$

Therefore, $\rho=+f$ corresponds to the upper material slipping with respect to lower solid in the direction of positive $x$, and $\rho=-f$ indicates slip in the opposite direction. However, it is generally not possible to predict the direction of slip without solving a specific problem because the sign of $\dot{C}$ is not known.

The singular fields corresponding to the root $0<\lambda_{0}<1$ of (3.10) which had to be discarded in contact problems can be present in crack problems because the boundary conditions in an adhesion zone are less stringent than those in a stick zone. The major difference is, of course, that the normal tractions may be tensile in the adhesion zone and the tangential tractions are not restricted by a friction law. The 
conditions that remain to be considered for the transition from adhesion to slip are (1.9) to (1.11). Using (3.18) to (3.20) we obtain

$$
\begin{aligned}
& |\rho|=f \\
& C \beta \leq 0 \\
& \operatorname{sgn}(\rho)=\operatorname{sgn}(\dot{h})=-\operatorname{sgn}(\dot{C}) .
\end{aligned}
$$

Thus $\rho=f$ again corresponds to a positive direction of slip and $\rho=-f$ to negative.

Unless $\beta=0$, an interface crack cannot have open tips, and the two faces of a crack must be in contact near the tips $[3,10,11]$. This is due to the fact that no direct transition from adhesion to separation is possible in crack problems for $\beta \neq 0$. It can also be reasoned [9] that slip in the contact zones must take place upon loading regardless of the value of the friction coefficient. Consider the case of $\beta>0$. Then according to (3.40) we have $C \leq 0$. Suppose that the loading of the body with an interface crack is started from zero level and increased monotonically. It can be conjectured then that $\dot{C}<0$, because $C$ can be viewed as a stress intensity factor which must clearly increase in absolute value as the level of loading is raised. Thus from (3.41) we have $\rho>0$, also $\rho \beta>0$ and consequently $0<\lambda_{0}<\frac{1}{2}$ by (3.10). Therefore the singularity at the crack tip is weaker than inverse square root. The same conclusion can also be reached for $\beta<0$. Suppose next that the load level is eventually decreased. It is clear that some back slip must start immediately because a stick zone cannot change directly to a separation zone. The only question is whether the back slip extends immediately to the tip of the crack, or a zone involving back slip propagates toward the end of the crack. In the first case, the singularity at the crack tip would be stronger than inverse square root $\left(\frac{1}{2}<\lambda_{0}<1\right)$; in the second, the singularity would be locked in until the back slip reaches the tip because the singularity cannot change in the absense of some new discontinuities.

\section{Transition from stick to separation}

The placement of the coordinate axes in relation to the solids and the two zones is shown in Figure 1c. The boundary conditions to be enforced are (3.1) to (3.4) and $(2.5)$ to $(2.8)$. The determinant is in this case $[2,12]$

$$
\Delta(\lambda)=\sin ^{2} \lambda \pi\left(\cos ^{2} \lambda \pi+\beta^{2} \sin ^{2} \lambda \pi\right) .
$$

The roots of (4.1) satisfying

$$
\sin \lambda \pi=0
$$

lead to elastic fields that enter the asymptotic analysis in a trivial way. Thus

$$
\begin{array}{ll}
x>0: & N(x)=T(x)=0 \\
x<0: & g(x)=0 .
\end{array}
$$


The root $\lambda=0$, for instance, yields the constant stresses

$$
\begin{array}{ll}
\sigma_{x x}^{(1)}=A(1-\alpha) ; & \sigma_{x y}^{(1)}=\sigma_{y y}^{(1)}=0 \\
\sigma_{x x}^{(2)}=A(1+\alpha) ; & \sigma_{x y}^{(2)}=\sigma_{y y}^{(2)}=0 .
\end{array}
$$

The other roots of (4.1) that satisfy

$$
\cos ^{2} \lambda \pi+\beta^{2} \sin ^{2} \lambda \pi=0
$$

are generally complex. Such complex roots lead to elastic fields that contain as multipliers the terms $\cos (\eta \log r)$ or $\sin (\eta \log r)$ where $\eta$ is the imaginary part of $\lambda$. These multipliers carry over into the expressions for the gap $g(x)$ and the tractions $N(x)$ and $T(x)$, and these quantities change sign an infinite number of times in the vicinity of the transition point. Consequently, neither the inequality $g(x) \geq 0$ in the separation zone nor $N(x) \leq 0$ in the stick zone can be satisfied. The conclusion we reach, therefore, is that the stick zone cannot change directly to a separation zone, and that there must always be an intervening slip zone, unless the combination of materials is special and $\beta=0$. The same conclusion can also be reached for an interface crack [9] which must generally have closed tips with slipping contact zones.

Oscillating singularities also arise at the vertices of two wedges that are bonded to each other [13]. If the bond is viewed as a stick zone, again the inequality (1.14) cannot be satisfied. Therefore we must conclude that, if two wedges are pressed together, some slip must take place near the vertices for wedge angles and combinations of materials such that Bogy's results [13] predict oscilating singularities.

The combinations of materials such that $\beta=0$, which includes identical materials and pairs of incompressible materials, is special because all roots of (4.1) are real.

The roots of

$$
\cos \lambda \pi=0
$$

lead to the following elastic fields:

$$
\begin{aligned}
& 2 \mu_{1} u_{r}^{(1)}=\frac{1}{2(1-\lambda)} r^{1-\lambda}\left\{C\left[\left(\kappa_{1}-1+\lambda\right) \cos \lambda \theta-\lambda \cos (2-\lambda) \theta\right]\right. \\
& \left.+D\left[-\left(\kappa_{1}-1+\lambda\right) \sin \lambda \theta+(2-\lambda) \sin (2-\lambda) \theta\right]\right\} \\
& 2 \mu_{1} u_{\theta}^{(1)}=\frac{1}{2(1-\lambda)} r^{1-\lambda}\left\{C\left[-\left(\kappa_{1}+1-\lambda\right) \sin \lambda \theta+\lambda \sin (2-\lambda) \theta\right]\right. \\
& \left.+D\left[-\left(\kappa_{1}+1-\lambda\right) \cos \lambda \theta+(2-\lambda) \cos (2-\lambda) \theta\right]\right\} \\
& \sigma_{r r}^{(1)}=\frac{1}{2} r^{-\lambda}\{C[(2+\lambda) \cos \lambda \theta-\lambda \cos (2-\lambda) \theta] \\
& +D[-(2+\lambda) \sin \lambda \theta+(2-\lambda) \sin (2-\lambda) \theta]\} \\
& \sigma_{r \theta}^{(1)}=\frac{1}{2} r^{-\lambda}\{C[\lambda \sin \lambda \theta+\lambda \sin (2-\lambda) \theta]+D[\lambda \cos \lambda \theta+(2-\lambda) \cos (2-\lambda) \theta]\} \\
& \sigma_{\theta \theta}^{(1)}=\frac{1}{2} r^{-\lambda}\{C[(2-\lambda) \cos \lambda \theta+\lambda \cos (2-\lambda) \theta] \\
& +D[-(2-\lambda) \sin \lambda \theta-(2-\lambda) \sin (2-\lambda) \theta]\} .
\end{aligned}
$$


The results for the upper solid are obtained by simply replacing $\mu_{1}$ with $\mu_{2}$ and $\kappa_{1}$ with $\kappa_{2}$. Furthermore,

$$
\begin{array}{ll}
x>0: & N(x)=C r^{-\lambda}, \quad T(x)=D r^{-\lambda} \\
x<0: & g(x)=K^{*} C r^{1-\lambda} \sin \lambda \pi \\
& K^{*}=\frac{1}{4(1-\lambda)}\left(\frac{\kappa_{1}+1}{\mu_{1}}+\frac{\kappa_{2}+1}{\mu_{2}}\right)>0 .
\end{array}
$$

A singularity corresponding to $\lambda=\frac{1}{2}$ is again precluded in contact problems by the inequalities (1.7) and (1.14). Thus the leading term in the expansion corresponds to $\lambda=-\frac{1}{2}$ for which the inequalities (1.7), (1.14), and (1.15) yield

$$
C \leq 0, \quad|D|<-f_{s} C \text {. }
$$

A singularity with $\lambda=\frac{1}{2}$ is of course possible in crack problems.

\section{Conclusion}

A remaining possibility is that there are logarithmic singularities in stress at the transition points. This is due to the fact [13] that in all three cases, as seen from (2.9), (3.9), and (4.1)

$$
\Delta(0)=0, \quad \frac{d \Delta(0)}{d \lambda}=0
$$

It was called to our attention by Sinclair [14], however, that (5.1) and (5.2) are not sufficient for the appearance of logarithmic singularities under the homogeneous boundary conditions considered, and that higher derivatives of $\Delta(\lambda)$ may have to vanish depending on the rank of the coefficient matrix at $\lambda=0$. The logarithmic singularities can be probed using complex potentials of the form $z \log z$. This was done, and it was found that all coefficients multiplying the potentials vanished under the homogeneous boundary conditions imposed. Thus there are no logarithmic singularities in stress at the three transitions considered; this conclusion is in agreement with the general results of Dempsey and Sinclair [15].

In summary, the following conclusions were reached when the pertinent inequalities are incorporated in the asymptotic analysis of contact and crack problems:

1. Regardless of the level of friction, no singularities can appear at any of the possible transitions in contact problems.

2. The contact pressure must vanish at the transition from a slip to a separation zone in contact problems.

3. Unless the combination of materials is special and $\beta=0$, no direct transition from a stick to a separation zone is possible, and there must be an intervening slip zone.

4. Again excluding the special case $\beta=0$, an interface crack must have closed tips, and a contact zone with slip separates the adhesion zone from the open part of the crack. 


\section{Acknowledgement}

We are indebted to Professor Sinclair of Carnegie-Mellon University for enlightening us on the point that logarithmic singularities are not necessarily possible when equations (5.1) and (5.2) are satisfied, and for sending us an advance copy of reference [15]. One of the authors (M.C.) is also pleased to acknowledge the support by the National Science Foundation of the United States under the grant ENG 77-25032.

\section{REFERENCES}

[1] Williams, M. L., Stress singularities resulting from various boundary conditions in angular corners of plates in extension. J. appl. Mech. 19 (1952) 526-528.

[2] Williams, M. L., The stress around a fault or crack in dissimilar media. Bull. seism. Soc. Am. 49 (1959) 199-204.

[3] Comninou, M., The interface crack. J. appl. Mech. 44 (1977) 631-636.

[4] Goodman, L. E., Contact stress analysis of normally loaded rough spheres. J. appl. Mech. 29 (1962) 515-522.

[5] Dundurs, J. and Stippes, M., Role of elastic constants in certain contact problems. J. appl. Mech. 37 (1970) 965-970.

[6] Dundurs, J., Properties of elastic bodies in contact. The Mechanics of the Contact Between Deformable Bodies, edited by A. D. de Pater and J. J. Kalker, Delft University Press 1975, 54-66.

[7] Gdoutos, E. E. and Theocaris, P. S., Stress concentrations at the apex of a plane indenter acting on an elastic half plane. J. appl. Mech. 42 (1975) 668-692.

[8] Comninou, M., Stress singularity at a sharp edge in contact problems with friction. Z. angew. Math. Phys. 27 (1976) 493-499.

[9] Comninou, M., Interface crack with friction in the contact zone. J. appl. Mech. 44 (1977) 780-781.

[10] England, A. H., A crack between dissimilar media. J. appl. Mech. 32 (1965) 400-402.

[11] Malyshev, B. M. and Salganik, R. L., The strength of adhesive joints using the theory of fracture. Int. J. Fracture Mech. 1 (1965) 114-128.

[12] Bogy, D. B., On the plane elastostatic problem of a loaded crack terminating at a material interface. J. appl. Mech. 38 (1971) 911-918.

[13] Bogy, D. B., Two edge-bonded elastic wedges of different materials and wedge angles under surface tractions. J. appl. Mech. 38 (1971) 377-386.

[14] Sinclair, G. B., Private communication.

[15] Dempsey, J. P. and Sinclair, G. B., On the stress singularities in the plane elasticity of the composite wedge, Report SM78-1, Carnegie-Mellon University. 